Introduction and Objectives Patients with pulmonary arteriovenous malformations (PAVMs) are difficult to assess for anaesthetic risks. Generally, they display well-preserved exercise tolerance, yet may have very low oxygen saturation due to their anatomical intrapulmonary right-to-left shunts. During pre-operative assessments in the general population, anaerobic threshold and peak $\mathrm{VO}_{2}$, measured by cardiopulmonary exercise testing (CPET), are increasingly recommended to identify high-risk patients, and appropriately plan post-operative management. For example, "high-risk" for major abdominal surgery has been suggested as an anaerobic threshold $<11 \mathrm{ml}$

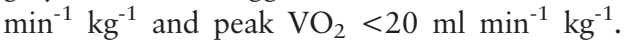

Methods In order to evaluate "pre-operative" risk categories for PAVM patients, anaerobic threshold and peak $\mathrm{VO}_{2}$, measured by ethically approved research cardiopulmonary exercise tests, were evaluated.

Results 26 PAVM patients underwent research CPET evaluations between April 2011-May 2017. Their median age was 57 years (interquartile range (IQR): 42-66). 16 (61.5\%) were male. The median oxygen saturation $\left(\mathrm{SaO}_{2}\right)$ was $92 \%$ (IQR: 88-95) and median haemoglobin $15.6 \mathrm{~g} / \mathrm{dl}$ (IQR: 14.2-16.6). Overall, the PAVM group achieved a median 92\% of the predicted maximum work (IQR: 67-106), anaerobic threshold ranged from 7.6-24.5 ml min $\mathrm{kg}^{-1}$ (median: 12.35; IQR: 9.5-17.35), and peak $\mathrm{VO}_{2}$ ranged from $11.2-45.5 \mathrm{ml} \mathrm{min}{ }^{-1}$ $\mathrm{kg}^{-1}$ (median: 19.8; IQR: 16.7-28.4). Anaerobic threshold placed $11 / 26(42.3 \%)$ in the suggested high-risk category for major abdominal surgery. In this group, the anaerobic threshold ranged from 7.6-10.8 ml min ${ }^{-1} \mathrm{~kg}^{-1}$. Similarly, peak VO2 placed 14/26 (53.8\%) in a high-risk category. Their peak $\mathrm{VO}_{2}$ ranged from $11.2-16.5 \mathrm{ml} \mathrm{min}^{-1} \mathrm{~kg}^{-1}$. There was full concordance between the categories determined by the 2 measurements. Notably, 6 patients were retested 3-31 months after embolization treatment resulting in increased $\mathrm{SaO}_{2}$. However, there was no increase in anaerobic threshold or peak $\mathrm{VO}_{2}$, and the 3 patients from this group initially in a higher risk category remained.

Conclusion Anaerobic threshold and peak $\mathrm{VO}_{2}$ suggest high proportions of PAVM patients are in a high-risk pre-operative risk category. The data suggest an important role for anaesthetic assessments. Noting that 1 in 2600 people are estimated to have PAVMs, further study is recommended to develop appropriate clinical guidance, and allocate resources to optimise care.

\section{P173 PROGNOSTIC FACTORS FOR SURVIVAL IN IDIOPATHIC PULMONARY ARTERIAL HYPERTENSION}

CR Popplewell, A Greenhalgh, PA Corris. Freeman Hospital, Newcastle-upon-Tyne, UK

\subsection{6/thoraxjnl-2017-210983.315}

Introduction Idiopathic pulmonary arterial hypertension (IPAH) is rare with an estimated prevalence of 5.9 cases/million population. ${ }^{1}$ Untreated, prognosis is poor with one year survival $68 \% .^{2}$ The ESC/ERS guidelines' risk assessment tool estimates one year mortality. ${ }^{1}$ This tool was used to assess the number of green prognostic factors and observe survival.

Methods Patients were identified retrospectively from a cohort of incident IPAH patients. Prognostic factors of WHO functional class, 6MWT and NT-proBNP were selected at 0 (baseline), 6 and 12 months. The number of green prognostic factors at baseline was compared with 6 and 12 months and survival observed.

Results 28 patients were identified; 11 male (39\%), 17 female (61\%), mean age at diagnosis 65 years (range 42-85). At baseline $19(68 \%)$ patients had no green prognostic factors compared with $12(43 \%)$ at 6 months $(\mathrm{p}=0.0045)$ and $11(39 \%)$ at 12 months $(p=0.002)$. At baseline $8 \quad(29 \%)$ had 1 green prognostic factor compared with $5(18 \%)$ at 6 months $(\mathrm{p}=0.08)$ and $10(36 \%)$ at 12 months $(\mathrm{p}=0.17)$. At baseline 1 (3\%) had 2 green prognostic factors compared with 11 (39\%) at 6 months $(\mathrm{p}=<0.0001)$ and $7(25 \%)$ at 12 months $(\mathrm{p}=0.006)$. No patients had 3 green prognostic factors. The number of patients with 0 red prognostic factors increased from $13(46 \%)$ at baseline to $20(71 \%)$ at 12 months $(\mathrm{p}=0.005)$. At one year all patients survived.

Conclusions There was a statistically significant increase $(\mathrm{p}=<0.05)$ in the number of patients with two green (low risk of mortality) prognostic factors at 6 and 12 months and patients with zero red (high risk) prognostic factors at 12 months, compared with baseline. A concurrent statistically significant reduction in number of patients with no green prognostic factors was seen. These suggest decreased one year mortality. One year survival between patients with 0,1 or 2 green prognostic factors was identical. Patients are being followed for long term survival.

\section{REFERENCES}

1. ESC/ERS. 2015 ESC/ERS Guidelines for the diagnosis and treatment of pulmonary hypertension. Eur Heart J 2016;37:67-119.

2. D'Alonzo GE. Survival in patients with primary pulmonary hypertension: Results from a National Prospective Registry. Ann Intern Med 1991;115(5):343-9.

\section{P174 A MULTICENTER, RETROSPECTIVE STUDY INTO EARLY MORTALITY IN ACUTE PULMONARY EMBOLISM}

${ }^{1} \mathrm{NM}$ Batt, ${ }^{1} \mathrm{~A}$ Radford, ${ }^{2} \mathrm{~K}$ Milinis, ${ }^{1} \mathrm{~K}$ Saraya. ${ }^{1}$ North West Thames NHS Trust, London, UK; ${ }^{2}$ Imperial Healthcare Trust, London, UK

\subsection{6/thoraxjnl-2017-210983.316}

Introduction and Objectives Prognostic accuracy and clinical utility of the Pulmonary Embolism Severity Index (PESI) \& simplified PESI (sPESI) at predicting very early mortality $(<7$ days) has not been previously investigated. We use this time frame to suggest outpatient imaging could be used to confirm a suspected pulmonary embolism (PE).

Methods A retrospective chart review of adult patients $(\geq 18$ years), who presented to two emergency departments, January 2013 - December 2015, with symptomatic pulmonary embolism (PE) confirmed on computed tomography pulmonary angiogram was carried out. Demographic and clinical parameters were recorded. PESI and sPESI scores were calculated and grouped into classes. Patients were followed up to 90 days. The primary outcome was death at 1, 3, 7, 30 and 90 days. Results Two hundred and eighty eight patients were eligible for inclusion. Mean age was 63 years (SD 18) and 51\% were male. Twenty-two patients died during the follow-up period. PE was attributed to the cause of death in 59\%. There was a significant correlation between PESI risk classes (I to V) and death at $3,7,30$ and 90 days $(\mathrm{p}<0.01)$ but not day 1 . No deaths occurred in the low risk sPESI class and no deaths occurred in PESI Class III. The discriminatory ability for each study end-point expressed as the area under the ROC curve was high (AUROC $\geq 0.8, \mathrm{p}<0.001$ ) for both PESI and sPESI. 